\title{
Avaliação cognitiva de pacientes em cuidados paliativos: estudo piloto*
}

\author{
Cognitive Assessment for Patients in Palliative Care: Pilot Study \\ Evaluación cognitiva de los pacientes en cuidados paliativos: un estudio piloto
}

Silvia Patricia Fernandes Coelho a

DOI: https://doi.org/10.11144/Javeriana.ie20-1.acpc

Universidade Católica Portuguesa, Portugal

Instituto de Ciências da Saúde, Portugal

sfcoelho@porto.ucp.pt

Recepção: 29 Fevereiro 2016

Cintia Pinho-Reis Aprovação: 01 Dezembro 2016

Fundação Fernando Pessoa do Hospital, Portugal

Universidade Fernando Pessoa, Portugal

Manuel Luis Vilas Capelas

Universidade Católica Portuguesa, Portugal

Instituto de Ciências da Saúde, Portugal

Luis Sá

Universidade Católica Portuguesa, Portugal

Instituto de Ciências da Saúde, Portugal

\section{Resumo:}

Objetivo: avaliar o estado cognitivo dos pacientes com necessidades paliativas, internados numa unidade de convalescença, através da utilização do instrumento, Escala de Pfeiffer. Material e métodos: estudo descritivo, observacional e transversal, realizado em 23 pacientes internados numa unidade de convalescença, em Portugal, no período 18 de fevereiro e 5 de março de 2014. A avaliação cognitiva foi efetuada com recurso à Escala de Pfeiffer-Versão Portuguesa. A análise estatística foi realizada com metodologia descritiva e analítica. Resultados: mais de $50 \%$ dos inquiridos erraram as questões que solicitavam o número de telefone e morada (82,6\%), conta de subtração (60,9\%) e o nome do anterior Presidente da República (56,5\%). Pacientes com alterações cognitivas têm uma mediana de 83 (66-87 anos). Conclusão: o estudo sugere que o uso da Escala de Pfeiffer constitui-se um instrumento adequado para a avaliação do estado cognitivo, dos pacientes com necessidades paliativas.

Palavras-chave: cuidados paliativos, determinação de necessidades de cuidados de saúde, cognição, pacientes internados, cuidados de enfermagem.

\section{Abstract:}

Aim: To assess cognitive status of patients with palliative needs, admitted to a convalescent unit, using the instrument, Pfeiffer Scale. Material and Methods: It is a descriptive, observational and cross-sectional study conducted in 23 patients admitted to a convalescent unit in Portugal, between February 18 and March 5, 2014. The cognitive assessment was performed using the Scale Pfeiffer-Portuguese version. Statistical analysis was performed with descriptive and analytical methodology. Results: Over $50 \%$ of respondents missed questions requesting the phone number and address $(82.6 \%)$, subtraction account $(60.9 \%)$ and the name of the former President of the Republic (56.5\%). Patients with cognitive impairments have a median of 83 (66-87anos). Conclusion: The study suggests that use of Pfeiffer Scale constitutes an appropriate tool for assessing the cognitive state of patients with palliative needs.

Keywords: palliative care, needs assessment, cognition, inpatients, nursing care.

\section{Resumen:}

Objetivo: evaluar el estado cognitivo de pacientes con necesidades paliativas, admitidos en una unidad de convalecencia, utilizando la Escala de Pfeiffer. Material y métodos: estudio descriptivo, observacional y transversal realizado en 23 pacientes ingresados en una unidad de convalecencia en Portugal, entre el 18 de febrero y el 5 de marzo de 2014. Se realizó la evaluación cognitiva

\section{Autor notes}


utilizando la Escala Pfeiffer versión portuguesa. El análisis estadístico se realizó con metodología descriptiva y analítica. Resultados: más del 50\% de los participantes erró en las preguntas que solicitaban el número de teléfono y domicilio (82,6\%), la operación de sustracción (60,9\%) y el nombre del anterior presidente de la República (56,5\%). La mediana de edad de los pacientes con alteraciones cognitivas era de 83 (66-87 años). Conclusiones: este estudio sugiere que el uso de la Escala de Pfeiffer constituye una herramienta apropiada para evaluar el estado cognitivo de los pacientes con necesidades paliativas.

Palabras clave: cuidados paliativos, evaluación de necesidades, cognición, pacientes internos, atención de enfermería.

\section{Introdução}

Os cuidados paliativos visam promover o bem-estar e a qualidade de vida dos pacientes e famílias que enfrentam problemas decorrentes de uma doença incurável e/ou grave e com prognóstico limitado, através da prevenção e alívio do sofrimento físico, psicológico, social e espiritual recorrendo à identificação precoce e tratamento rigoroso da dor, bem como, outros problemas físicos, psicossociais e espirituais [1,2].

Atualmente as pessoas vivem mais anos, o que as predispóe aos efeitos da senescência, a maior fragilidade e vulnerabilidade e consequentemente ao aparecimento de mais doenças. Por isso torna-se imprescindível dar respostas ao nível da saúde, congruentes e sensíveis a estas necessidades emergentes da população [3].

A mudança no perfil populacional, associada ao aumento do índice de dependência dos idosos e às mudanças de caráter económico e social, determinam transformações na prevalência e incidência das doenças crónicas gerando a necessidade de uma adequada integração e articulação entre cuidados curativos e cuidados paliativos, através de uma transição progressiva para estes últimos, quando a evolução da situação clínica se justificar, e se for do desejo do paciente e da família $[4,5]$.

Atendendo às mudanças demográficas, uma população cada vez mais envelhecida e estimando-se que 5\% a $10 \%$ dos idosos possuem alguma perda da capacidade cognitiva, mais prevalente nos com 80 ou mais anos, é fulcral que os profissionais desenvolvam intervenções e competências para minimizar, e responder às necessidades destas populações [6,7].

O recurso a instrumentos que possibilitem identificar, avaliar e diagnosticar as alterações cognitivas são determinantes para a reversibilidade da situação ou mesmo para atenuar a deterioração clínica contribuindo para uma melhor qualidade de vida [7].

A opção pela Escala de Pfeiffer, validada para a versão portuguesa, justifica-se visto ser uma escala já implementada por algumas comunidades científicas, na sua prática assistencial em cuidados paliativos.

A justificação do estudo baseia-se na premência do tema, visto a importância de se incorporarem as decisões, as preferências e expectativas dos pacientes face ao plano terapêutico respeitando desta forma, a sua autodeterminação, autonomia e dignidade. Assim, torna-se fundamental a avaliação do estado cognitivo dos pacientes, para permitir definir um plano avançado de cuidados, no controlo de sintomas baseado nos desejos, intenções dos pacientes e satisfação das suas necessidades, e responder atempadamente às suas opções [8].

O estudo teve como objetivo avaliar o estado cognitivo dos pacientes com necessidades paliativas, internados numa unidade de convalescença, através da utilização do instrumento, Escala de Pfeiffer.

\section{Materiais e métodos}

Delineámos um estudo descritivo, observacional e transversal, realizado junto de pacientes internados numa unidade de convalescença, da rede nacional de cuidados continuados integrados, do norte de Portugal, no período de 18 de fevereiro a 5 de março de 2014.

Esta unidade foi escolhida por ser destinada a pacientes/pessoas que apresentam uma doença crónica, ou se encontram em fase de recuperação de um processo agudo com perda de autonomia potencialmente recuperável. Nesta tipologia de unidades existem pacientes numa fase de transição, entre cuidados ditos curativos para cuidados de suporte ou mesmo cuidados paliativos, segundo um planeamento diferenciado, 
ao nível do controlo de sintomas, tratamento da dor e intervenções no alívio do sofrimento, e outra sintomatologia subjacente, permitindo verificar a adequação da escala, relativamente a pacientes com necessidades de cuidados paliativos.

Os pacientes internados na unidade possuem uma grande heterogeneidade clínica, sendo favorável segundo os investigadores para o estudo, pois os cuidados paliativos não se destinam apenas a pacientes oncológicos mas também a pacientes com patologias ameaçadoras da vida, com doenças crónicas que acarretam um enorme sofrimento, e a pacientes que enfrentam problemas decorrentes de uma doença incurável e/ou grave e com prognóstico limitado $[9,10,11]$.

O período em que foi realizado o estudo, ocorre numa fase em que um dos investigadores se encontrava na unidade, a desenvolver um estudo de investigação, e necessitava de avaliar o estado cognitivo dos pacientes. $\mathrm{O}$ período estendeu-se a 20 dias visto que a previsão de internamento nestas unidades está limitada até 30 dias consecutivos de internamento. A avaliação do estado cognitivo foi efetuada com recurso à Escala de Pfeiffer versão portuguesa.

A Escala de Pfeiffer é uma das ferramentas existentes para avaliação da função cognitiva. Através de heteroavaliação e de uma forma simples, deteta a presença de comprometimento cognitivo. Avalia a memória de curto prazo e faculta uma orientação sobre a memória de longo prazo, bem como, informações sobre eventos diários e cálculo da capacidade [12].

Esta escala está indicada em qualquer pessoa/paciente que requeira uma avaliação da sua capacidade cognitiva [13]. Tem como pontos fortes, ser um instrumento validado em países com forte desenvolvimento nos cuidados paliativos, sendo amplamente utilizado por profissionais de vários sistemas de Saúde (Espanha, Inglaterra e Canadá), sobretudo por ser simples, de rápida aplicação ( 5 minutos), com uma sensibilidade próxima de $70 \%$ e especificidade de $95 \%$ [13].

A sua validação para Portugal foi realizada através de um estudo de tradução e a adaptação cultural para o português europeu, que resultou na análise/validação por peritos e aplicação a 302 pessoas ( 147 residentes em lares e 155 a usufruírem de centros de dia), verificando-se que é útil para obter informações significativas que auxiliem a focar as intervenções e a promover estratégias sensíveis às necessidades de cada pessoa [14]. A versão, carateriza-se por 10 perguntas (tabela 1). $O$ ponto de corte situa-se em 4 ou mais respostas erradas.

TABELA 1

Itens da Escala de Pfeiffer

\begin{tabular}{|l|}
\hline \multicolumn{1}{|c|}{ Itens } \\
\hline 1. Data \\
2. Dia da semana \\
3. Nome do local onde está, no momento \\
4. Número de telefone \\
5. Morada \\
6. Idade \\
7. Data de nascimento \\
8. Nome do atual presidente da República \\
9. Nome do anterior presidente da República \\
10. Nome completo da mãe \\
11. Contagem decrescente de 3 em 3, a partir de 20 \\
\hline
\end{tabular}

Fuente: elaboración propia 
O estudo foi aplicado a uma amostra acidental 23 pacientes (todos os que estavam internados no dia que foi efetuada a recolha de dados e que coincidia com a lotação máxima da unidade). Os critérios de inclusão foram: pacientes internados na unidade de convalescença, terem mais de 18 anos, capacidade de comunicar verbalmente, entenderem a língua portuguesa e aceitarem participar no estudo. Após a explicação do objetivo do estudo e da respetiva escala, e solicitação do consentimento para aplicar a mesma, aos pacientes que correspondiam aos restantes critérios, todos os pacientes aceitaram participar pelo que amostra coincide com o total de pacientes internados na unidade. Definiram-se como variáveis de caraterização dos pacientes o género, idade, escolaridade, diagnóstico e comorbilidades (tabela 2).

TABELA 2

Características dos pacientes $(\mathrm{n}=23)$

\begin{tabular}{|c|c|c|}
\hline Variável & $\mathbf{n}$ & $\%$ \\
\hline \multicolumn{3}{|l|}{ Sexo } \\
\hline Feminino & 14 & 60,9 \\
\hline Masculino & 9 & 39,1 \\
\hline \multicolumn{3}{|l|}{ Idade (anos) } \\
\hline $54-64$ & 3 & 13,0 \\
\hline $65-74$ & 6 & 26,1 \\
\hline $75-84$ & 11 & 47,9 \\
\hline $85-94$ & 3 & 13,0 \\
\hline \multicolumn{3}{|l|}{ Escolaridade } \\
\hline Analfabeto & 4 & 17,4 \\
\hline $1^{\circ}$ ciclo (1-4 anos) & 15 & 65,3 \\
\hline $2^{\circ}$ ciclo (5-6 anos) & 1 & 4,3 \\
\hline $3^{\circ}$ ciclo (7-9 anos) & 2 & 8,7 \\
\hline Licenciatura & 1 & 4,3 \\
\hline \multicolumn{3}{|l|}{ Doença principal } \\
\hline Status pós-cirurgia ortopédica & 11 & 47,8 \\
\hline AVC & 6 & 26,1 \\
\hline Outros & 6 & 26,1 \\
\hline \multicolumn{3}{|c|}{ N. ${ }^{\circ}$ de co morbilidades } \\
\hline 1 & 2 & 8,7 \\
\hline $2-3$ & 5 & 21,7 \\
\hline $4-5$ & 10 & 43,5 \\
\hline 6-7 & 6 & 26,1 \\
\hline
\end{tabular}

Fuente: elaboración propia

A recolha dos dados foi realizada na unidade do paciente, por entrevista, com especial atenção à comunicação não-verbal. No início, os pacientes foram informados sobre a finalidade da escala de Pfeiffer e do estudo para obter o seu consentimento livre e esclarecido quanto à participação no mesmo, de acordo com os princípios éticos da Declaração de Helsinki. A participação foi voluntária, obtendo-se o respetivo consentimento e atendeu-se ao respeito pela confidencialidade dos dados, e intimidade dos participantes. A escala foi aplicada sempre pelo mesmo investigador e foi solicitada a colaboração de familiares para validação de algumas das respostas. 
A análise dos dados teve como referencial metodológico, a análise estatística das respostas da Escala de Pfeiffer, visto que pretendemos avaliar o estado cognitivo, de pacientes com necessidades de cuidados paliativos, internados numa unidade de convalescença.

$\mathrm{Na}$ análise estatística foi avaliada a normalidade das diversas distribuições para a tomada de decisão quanto à escolha de testes paramétricos ou não paramétricos. Os testes não paramétricos utilizados foram o de Binomial, Qui-quadrado (sexo versus défice cognitivo; diagnóstico de AVC versus défice cognitivo), Kruskall-Wallis (diagnóstico versus défice cognitivo). Do conjunto de testes paramétricos foi utilizado o t-Student (idade versus défice cognitivo) e a correlação de Pearson (idade versus $n .{ }^{\circ}$ de erros; n. ${ }^{\circ}$ de comorbilidades versus $n .^{\circ}$ de erros). Considerou-se, como significativo o valor de $\mathrm{p}<0,05$.

A avaliação do estado cognitivo foi realizada com o recurso à Escala de Pfeiffer e os dados analisados através da estatística descritiva e analítica, com a utilização do Software IBM Statistical Package for the Social Sciences (SPSS) statistics versus 22.0, Chicago Inc.

\section{Resultados}

A tabela 2 demonstra as características avaliadas dos pacientes. Quanto ao género 39,1\% eram homens (teste Binomial: $\mathrm{p}=0,405)$. No que respeita à idade, os pacientes apresentavam uma média de 75,4 $\pm 10,2$ e mediana de 77 anos (54,94 anos). Por outro lado, a maioria dos pacientes tinha apenas o $1^{\circ}$ ciclo de escolaridade, assim, como o diagnóstico principal era maioritariamente status pós-cirurgia ortopédica (47,8), seguido de AVC $(26,1 \%)$ e os restantes $26,1 \%$ abrangendo outros diagnósticos médico-cirúrgicos. Quanto às co morbilidades, os pacientes apresentavam uma média de 4,2 $\pm 1,7$ e mediana de 4 [1,2,3,4,5,6,7].

No que respeita ao número de erros (tabela 3), verificou-se uma média de 3,3 $\pm 1,5$ e mediana de 3 , com uma variação entre 0 e 6 erros. Correlacionando a idade com o número de erros, através do teste de correlação de Pearson (visto ambas as distribuições serem normais), obteve-se uma correlação moderada positiva $(\mathrm{r}=$ $0,454 ; \mathrm{p}=0,030)$.

TABELA 3

Pacientes por numero de erros no teste de Pfeiffer $(n=23)$

\begin{tabular}{|c|r|r|}
\hline N. $^{\mathbf{0}}$ de erros & \multicolumn{1}{|c|}{ n } & \multicolumn{1}{c|}{ \% } \\
\hline 0 & 1 & 4,3 \\
\hline 1 & 2 & 8,7 \\
\hline 2 & 4 & 17,4 \\
\hline 3 & 5 & 21,7 \\
\hline 4 & 7 & 30,4 \\
\hline 5 & 3 & 13,0 \\
\hline 6 & 1 & 4,3 \\
\hline
\end{tabular}

Fuente: elaboración propia

As perguntas em que mais de $50 \%$ dos inquiridos erraram foram as que solicitavam o número de telefone e a morada $(82,6 \%)$, a prova de subtração $(60,9 \%)$ e o nome do anterior presidente da República (56,5\%), Embora, não existiram substancialmente, percentagens significativas de erros na data (47,9\%) e na identificação do local onde estavam (39,1\%) (tabela 4). 
TABELA 4

Pacientes que erraram em cada um dos itens do teste de Pfeiffer $(\mathbf{n}=23)$

\begin{tabular}{|l|r|r|}
\hline \multicolumn{1}{|c|}{ Itens } & n & \% \\
\hline Data & 11 & 47,9 \\
\hline Dia da semana & 4 & 17,4 \\
\hline Nome do local onde está, no momento & 9 & 39,1 \\
\hline Número de telefone/morada & 19 & 82,6 \\
\hline N. $^{\circ}$ de anos/idade & 2 & 8,7 \\
\hline Data de nascimento & 0 & 0,0 \\
\hline Nome do atual presidente da República & 0 & 0,0 \\
\hline Nome do anterior presidente da República & 13 & 56,5 \\
\hline Apelido & 0 & 0,0 \\
\hline $\begin{array}{l}\text { Contagem decrescente de 3 em 3, iniciando em } \\
20\end{array}$ & 14 & 60,9 \\
\hline
\end{tabular}

Maioritariamente, (teste Binomial: $\mathrm{p}=0,011)$ os pacientes não apresentavam défice cognitivo $(78,2 \%$ = 18 pacientes) ou seja, tal só foi detetado em 21,8\% [5] dos pacientes. Analisando-se a possibilidade de existirem diferenças nas idades dos sujeitos com e sem alterações cognitivas, constatamos que os pacientes com alterações cognitivas têm uma média de 79,6 \pm 8,2 anos, mediana de 83 (66-87 anos), enquanto os pacientes sem alterações apresentam uma média de 74,3 \pm 10.5, mediana de 76,5 (54-94 anos). Pela aplicação do teste $\mathrm{t}$-Student (visto as distribuições serem normais) tais diferenças não são estatisticamente significativas $[\mathrm{t}(21)$ $=1,037 ; \mathrm{p}=0,311$.

Por outro lado, agrupando os pacientes em 3 categorias de diagnóstico principal (AVC | Status pós cirurgia | outros diagnósticos médicos) verificou-se através do teste de Kruskal-Wallis, que não existiam diferenças estatisticamente significativas, no número de erros entre os grupos $\left[\mathrm{KW}-\chi^{2}(2)=0,643 ; \mathrm{p}=0,725\right]$. Pela análise com o teste de qui-quadrado (correção de Monte Carlo) verificou-se que não existe relação estatística significativa entre o diagnóstico e o défice cognitivo $\left[\chi^{2}(2)=2,339 ; \mathrm{p}=0,460\right]$.

Numa outra perspetiva, avaliando a existência de alguma interdependência entre o défice cognitivo e ter ou não, como diagnóstico principal AVC, foi calculado o odds ratio (OR) que demonstrou não existirem diferenças nas proporções observadas nos dois grupos de situação clínica (OR = 2,333; IC95\%: 0,284-19,172). Também verificou-se não existir correlação estatisticamente significativa entre o número de erros na Escala de Pfeiffer e o número de co morbilidades $(\mathrm{r}=0,201 ; \mathrm{p}=0,357)$.

\section{Discussão}

O presente estudo demostrou que a maioria dos pacientes não apresenta défice cognitivo (78,0\%). Emerge assim que a escala permite efetuar a avaliação cognitiva dos pacientes, contudo urge paralelamente utilizar documentação que possibilite avaliar as necessidades e preferências dos pacientes pois irá garantir a continuidade dos cuidados. Também facilitará a colaboração e comunicação entre a equipa interdisciplinar, permitindo a atuação entre os vários elementos em conformidade com as decisões dos pacientes e ainda, o envolvimento e adesão ao plano terapêutico.

Este estudo corrobora com os achados de outros estudos realizados em pacientes com doença avançada pois os transtornos cognitivos estão entre as complicações psiquiátricas mais frequentes, e estão associados a uma elevada morbilidade e impacto sobre a tomada de decisão em relação à sua doença $[15,16]$.

A integração precoce nos cuidados paliativos pode otimizar o reconhecimento pela equipa e minimizar o impacto da auto consciencialização da deterioração cognitiva do paciente, e cuidadores na fase inicial e 
na gradual deterioração pela monotorização sistemática através da aplicação de instrumentos, atenuando o sentimento de perda, prevenindo e aliviando um sofrimento destrutível e por vezes intolerável $[17,18]$.

A avaliação contínua do estado cognitivo contribui para uma melhor avaliação e monitorização sistemática dos restantes sintomas, como dor, dispneia, delirium, agitação, depressão, astenia intensa ou caquexia, sendo que alguns sintomas requerem uma resposta atempada e eficaz, minimizando o peso destes, na vida dos pacientes $[19,20]$.

Nos pacientes, cujo défice cognitivo está presente, a equipa de saúde, em especial os enfermeiros devem reunir junto de todos os intervenientes, o maior conjunto de informações, que permita um processo de tomada de decisão centrado no paciente, incluindo a avaliação cuidada de todos os sintomas, no sentido do despiste, de situações complicadas e atuar assertivamente nas situações que possam ser corrigíveis. Deste modo, o objetivo é preservar a capacidade do paciente para uma tomada de decisão partilhada assim como, implementar precocemente intervenções e medidas para manter, e conservar as funções não afetadas [20,21].

Os cuidados prestados devem ser congruentes com as preferências, valores, expetativas de vida, dos pacientes mas fundamentalmente atendendo às necessidades individuais face à situação clínica [20], ao controlo de sintomas e outras patológicas já existentes ou recorrentes, como sinónimo de qualidade e satisfação dos pacientes [18,21,22].

Uma série de valores e princípios éticos, são reconhecidos por diversos especialistas em cuidados paliativos, nomeadamente, o valor da autonomia, a manutenção da dignidade, a necessidade de um plano individualizado e a envolvência do paciente na tomada de decisão [21,22,23].

O recurso a instrumentos de avaliação standardizados e validados, em pacientes com necessidades paliativas é fundamental para avaliar e monitorizar os problemas, para aferir cientificamente as necessidades e assim estabelecer-se um plano de cuidados personalizado. Estes permitem a garantia de cuidados básicos, uma triagem clínica, a avaliação da capacidade de participação do paciente na decisão clínica e a continuidade do plano de cuidados [24].

Atendendo a que a média das idades foi de 75,4 anos, com 4,2 e 4 de número médio de co morbilidade e mediana respetivamente, estamos perante sujeitos que se enquadram numa abordagem e filosofia dos cuidados paliativos, devendo estes cuidados serem integrados no plano terapêutico pois conforme supracitados irão exigir apoio específico, organizado e interdisciplinar através de cuidados personalizados, globais e especializados [22].

Estando despertos para os problemas decorrentes do envelhecimento, em que propicia o aumento da prevalência de patologia crónica e diversas outras incapacidades, da dependência de terceiros, assim como, o aumento da fragilidade e vulnerabilidade, a atuação dos profissionais deve inevitavelmente incluir a avaliação do estado cognitivo, o mais precocemente possível com o intuito de definir as prioridades, necessidades, expetativas dos pacientes perante a sua situação clínica, como um cuidado antecipatório ao declínio [25,26].

No estudo verificamos que não existem diferenças estatisticamente significativas entre as idades médias dos pacientes com alterações cognitivas e dos que não apresentam, o que torna fundamental identificar as habilidades, capacidades e competências intelectuais e cognitivas de cada paciente para a tomada de decisão pois trata-se de um processo, que é necessário perante a evidência da indispensabilidade de decidir e resolver problemas [15].

A decisão clínica deverá ser sempre baseada nos aspetos éticos, ou seja nas características da doença principal e grau de evolução da mesma, gravidade da situação, complicações adjacentes, opinião do paciente (se já antes, enquanto competente, a expressou devidamente), atitude do mesmo face aos cuidados de que é alvo, opinião dos profissionais que mais horas permanecem com o paciente, grau do controlo sintomático e conforto, opinião da família e a qualidade de vida e bem-estar do paciente [18].

Relativamente ao número de erros na escala de Pfeiffer e o diagnóstico verificámos que não há associação entre no número de erros e diagnóstico $(\mathrm{p}>0,05)$, prevalecendo diagnósticos como AVC, status pós cirurgia e outros diagnósticos médicos. Constámos ainda, que o diagnóstico de AVC não condiciona, nestes pacientes, 
a existência de alteração da função cognitiva pois independentemente, da patologia ser do foro neurológico ou não, é necessário avaliar sempre a função cognitiva para estabelecer metas e definir objetivos terapêuticos, fomentando o auto cuidado, a negociação e adesão ao regime terapêutico.

$\mathrm{O}$ uso de instrumentos de avaliação adequados permite aos profissionais associarem o seu corpo de conhecimentos, domínio de técnicas e competências, recorrendo a uma prescrição terapêutica baseada em evidência científica, a uma monitorização contínua dos diversos problemas, à articulação entre equipa e paciente/família que favorecem a prestação de cuidados especializados, individualizados, humanos e holísticos [27,28].

Da revisão bibliográfica emanou que embora as questões relacionadas com a doença (diagnóstico, prognóstico e tratamento) sejam importantes para os pacientes com doenças crónicas, complexas, avançadas e limitadoras da vida, as questões relacionadas à evolução da mesma, possíveis complicações, consequências e custos a nível individual e social, são descritos como os temas mais importantes [29].

Assim, a avaliação cognitiva de cada paciente é fundamental para a manutenção da sua auto-determinação em relação à progressão da doença bem como, determinar medidas preventivas e antecipatórias para que a equipa tenha conhecimento das decisões e preferências dos pacientes, em situações em que estes se encontrem impossibilitados de manifestar a sua vontade consciente, livre e esclarecida, no que concerne aos cuidados que desejam receber, ou não desejam receber [18].

A falta de intervenções preventivas, a alta taxa de mortalidade dos pacientes com défices cognitivos [7,30], a dificuldade da precisão de um diagnóstico devido à presença de sinais e sintomas pouco diferenciados e por vezes, desvalorização de algumas respostas como esquecimento, falta de atenção, desinteresse e falta de iniciativa, querer dos profissionais uma atenção rigorosa [7,31].

Nos pacientes sem alterações cognitivas, a avaliação cognitiva precoce, possibilita intervir antecipadamente e planear futuras intervenções e, a envolvência do paciente nas decisões e ainda permite, respeitar o seu princípio de autonomia [24].

O grau de envolvimento dos profissionais, o conhecimento das suas expetativas e preferências e a adequação às necessidades dos pacientes são aspetos facilitadores para um cuidado integral. $O$ paciente deve, sempre que reúna as condições cognitivas necessárias, estar envolvido nas decisões passíveis de serem tomadas, pelo que a utilização de um instrumento de avaliação como a Escala de Pfeiffer constitui-se de grande utilidade no planeamento avançado dos cuidados. Assim, evidencia-se como desafio para os profissionais de saúde, a adoção de um papel ativo, devendo ser discutidas e negociadas com o paciente, todas as intervenções.

Aauto e heteroavaliação dos problemas físicos, sociais, espirituais e psicológicos facilita a aplicação de medidas e tratamentos com o conhecimento dos pacientes e o consentimento dos mesmos, bem como, adequar as intervenções, proporcionando uma abordagem abrangente para colmatar as suas necessidades e uma conceção de intervenções para atingir metas como o conforto, o bem-estar e a qualidade de vida [32].

Os dados identificados no presente trabalho coadunam-se com os resultados de outros estudos [24,33], onde estes identificam a prioridade de avaliar a função cognitiva dos pacientes como uma atitude preditiva/ preventiva na progressão da doença, permitindo um melhor planeamento e introdução de novas metas e tratamentos, envolvendo o paciente nas decisões clínicas [24,33].

Ressalvando-se a concordância dos dados com a literatura, há que destacar o facto de o estudo ter sido desenvolvido em contexto de internamento, devendo o mesmo ser replicado para os cuidados domiciliários de forma, a aferir os resultados e dar respostas às atuais preferências dos pacientes com necessidades paliativas visto que $51 \%$ dos pacientes preferem morrer em casa [34] sendo o local privilegiado para um melhor atendimento [35].

Todas as equipas, independentemente do contexto devem atender às expectativas dos pacientes, relacionando as necessidades e preocupações tanto com a evolução da doença, como com o tratamento, ou mesmo com a morte. A evidência demostra que os profissionais de saúde devem estar atentos para a diversidade de preocupações dos pacientes e devem monitorizar constantemente as expectativas e 
necessidades, de forma a identificar, negociar e resolver criativamente e eficazmente estas situações, atuando segundo uma perspetiva integradora [35].

A identificação e avaliação do estado cognitivo é crucial para responder objetivamente às necessidades dos pacientes internados de forma, a não perderem a sua liberdade e autonomia. Estudos desenvolvidos na comunidade, revelam existir menor deterioração cognitiva do que os desenvolvidos junto de pacientes institucionalizados [36]. Assim, é necessário comprovar se a hospitalização/internamento é um fator de risco para aumentar a deterioração cognitiva, ou se devido a estes pacientes estarem sujeitos a uma maior fragilidade, é mais frequente [6].

\section{Conclusão}

A avaliação do estado cognitivo com recurso a instrumentos de avaliação estandardizados, tais como a Escala de Pfeiffer, permite responder ao desafio de prestar cuidados paliativos com qualidade e centrados nos pacientes e família.

Dado o tempo exíguo da pesquisa, a população ser pequena e heterogênea, em termos de idade, educação e condição clínica pode ter condicionado, resultados menos relevantes, pelo que se tornam necessários, no futuro, estudos mais alargados, comparando o contexto de internamento com o contexto domiciliário relativamente à identificação do estado cognitivo dos pacientes com necessidades paliativas.

\section{Conflito de interesses}

Os autores declaram não haver conflitos de interesse relacionados com este manuscrito.

\section{Financiamento}

Não existiu qualquer tipo de financiamento para a realização do estudo.

\section{Referências}

1. World Health Organization. WHO definition of palliative care [Internet]. 2009 [citado 2009 sep 19]. Disponível em: http://www.who.int/cancer/palliative/definition/en/

2. Silva M, Lima L. Participação do familiar nos cuidados paliativos oncológicos no contexto hospitalar: perspectiva de enfermeiros. Rev Gaúcha Enferm. 2014 dec;35(4):14-9.

3. Piers R, Pautex S, Curiale V, Curale V, Pfisterer M, Van Nes M-C, et al. Palliative care for the geriatric patient in Europe: Survey describing the services, policies, legislation, and associations. Z Gerontol Geriatr [Internet]. 2010 Dec [citado 2014 Dec 30];43(6):381-5. Disponível em: http://www.ncbi.nlm.nih.gov/pubmed/21103991

4. Capelas ML, Coelho SP. A morte e a boa morte hoje. Patient Care. 2013;18(197).

5. Vargas-Escobar LM. Marco para el cuidado de la salud en situaciones de enfermedad crónica. Investig Enferm Imagen Desarr [internet]. 2010;12(1):79-94. Disponível em: http://revistas.javeriana.edu.co/index.php/imagenydesar rollo/article/viewFile/1626/1048

6. Nancy E. Differentiating the three Ds: Delirium, dementia and depression. Medsurg Nurs. 2003;12(6):347-57.

7. Silva R, Santos S, Lourenço M, Coelho S, Sá L. Deterioração cognitiva no idoso: Revisão da literatura. Em:: Zutin TLM, Serva FM,orgs. Gerontologia: Olhares diversos sobre o envelhecimento. São Paulo: Arte \& Ciência; 2015. p. 203-25. 
8. van Soest-Poortvliet MC, van der Steen JT, de Vet HC, Hertogh CM, Deliens L, Onwuteaka-Philipsen BD. Comfort goal of care and end-of-life outcomes in dementia: A prospective study. Palliat Med. 2015;29(6):538-46. doi: 10.1177/0269216315570409

9. National Quality Forum. A national framework and preferred practices for palliative and hospice care quality. Washington: National Quality Forum; 2006.

10. National Consensus Project for Quality Palliative Care. Clinical practice guidelines for quality palliative care. 2nd ed. Pittsburgh: National Consensus Project for Quality Palliative Care; 2009.

11. Capelas M, Coelho S. Pensar a organização dos serviços de saúde. Cuidados Paliativos. 2014;1(1):17-26.

12. Pfeiffer $\mathrm{E}$. A short portable mental status questionnaire for the assessment of organic brain deficit in elderly patients. J Am Geriatr Soc. 1975;23(70):433-41.

13. Gobierno de Aragón-Departamento de Salud y Consumo. Programa de Atención a Enfermos Crónicos Dependientes. Zaragoza; 2006. p. 207-8.

14. Rodrigues RMC. Validação da versão em português europeu de questionário de avaliação funcional multidimensional de idosos. Rev Panam Salud Publica. 2008;23(2):109-15.

15. Becker R. Palliative care 2-exploring the skills that nurses need to deliver high-quality care. Nurs Times. 2009;105(14):18-20.

16. Mystakidou K, Parpa E, Galanos A, Vlahos L. Brief cognitive assessment of cancer patients: Evaluation of the MiniMental State Examination (MMSE) psychometric properties. Psycho-Oncology. 2007;16:352-7.

17. Addington-Hall J, Higginson I, editores. Palliative care for non cancer patients. Oxford: Oxford University Press; 2001.

18. Neto I. Cuidados paliativos em pessoas com demência avançada. Geriatrics. 2001;4(9):47-56.

19. Covinsky K, Yaffe K. Dementia, prognosis, and the needs of patients and caregivers. Ann Intern Med. 2004;140(7):573-5.

20. Domoto-Reilly K, Sapolsky D, Dickerson B. Mild Cognitive Impairment (MCI) stage of the frontotemporal dementias: Early diagnosis and management. Perspect Gerontol. 2014;19(2):57-64.

21. Sapeta P. Cuidar em fim de vida: o processo de interação enfermeiro-doente. Loures: Lusociência-Edições Técnicas e Científicas; 2011.

22. Radbruch L, Payne S, Bercovitch M, Caraceni A, Vlieger T De, Firth P, et al. White paper on standards and norms for hospice and palliative care in Europe: part 1. Eur J Palliat Care [Internet]. 2009;16(6):278-89. Disponível em: http://eprints.lancs.ac.uk/32714/

23. Fernandes L. Aspectos éticos e legais nos estados avançados de demência. Acta Médica Portuguesa. 2008;21:65-72.

24. Reys B, Bezerra A, Vilela A, Keusen A, Marinho V, De Paula E, Laks J. Diagnóstico De demência, depressão e psicose em idosos por avaliação cognitiva breve. Rev Ass Méd Bras. 2006;52(6):401-4.

25. Moreno-Monsiváis M, Muñoz-Rodríguez M, Interial-Guzmán M. Satisfacción con el manejo del dolor posoperatorio en pacientes hospitalizados. Aquichan. 2014;14(4):460-72.

26. Ferrell B, Connor SR, Cordes A, Dahlin CM, Fine PG, Hutton N, Leenay M, Lentz J, Person JL, Meier DE, Zuroski K; National Consensus Project for Quality Palliative Care Task Force Members. The national agenda for quality palliative care: The National Consensus Project and the National Quality Forum. J Pain Symptom Manage [Internet]. 2007 Jun [citado 2013 Fev 6];33(6):737-44. Disponível em: http://www.ncbi.nlm.nih.go v/pubmed/17531914

27. Neto IG. Princípios e filosofia dos cuidados paliativos. Em: Barbosa A, Neto IG, editores. Manual de cuidados paliativos. 2th ed. Lisboa: Faculdade de Medicina de Lisboa; 2010. p. 1-42.

28. Campo C. Cuidado que trasciende más allá de la muerte. Investig Enferm Imagen Desarr. 2013;(1):85-94.

29. Gomes B, Calanzani N, Curiale V, McCrone P, Higginson IJ. Effectiveness and cost-effectiveness of home palliative care services for adults with advanced illness and their caregivers. Cochrane Database Syst Rev. 2013;(6): CD007760. doi: 10.1002/14651858.CD007760.pub2 
30. Tsai M, Weng H, Chou S, Tsai C, Hung T, Su J. One-year mortality of elderly inpatients with delirium, dementia, or depression seen by a consultation-liaison service. Psychosomatics. 2012, 53(5):433-8.

31. Lach HW, Chang YP, Edwards D. Can older adults with dementia accurately report depression using brief forms? Reliability and validity of the Geriatric Depression Scale. Journal Gerontol Nursing. 2010, 36 (5):30-7.

32. Guerrero LL, Catalán AG. Variáveis biopsicossociais relacionadas à duração de hospitalização em idosos. Rev Lat Am Enfermagem. 2011;19(6):8 telas.

33. Molinari V., Chiriboga D., Branch LG, Greene J, Schonfeld L, Vongxaiburana E, Hyer, K. Influence of Mental Health Assessment on Prescription of Psychoactive Medication Among New Nursing Home Residents. Clin Gerontol [Internet]. 2013 Jan [citado 2014 Dec 30];36(1):33-45. Disponível em: http://www.tandfonline.co $\mathrm{m} /$ doi/abs/10.1080/07317115.2012.731475

34. Gomes B, Higginson IJ, Calanzani N, Cohen J, Deliens L, Daveson BA, et al. Preferences for place of death if faced with advanced cancer: a population survey in England, Flanders, Germany, Italy, the Netherlands, Portugal and Spain. Ann Oncol. 2012;23(8):1-11.

35. Gamboa-Bernal G. Atención domiciliaria de la persona anciana: una perspectiva bioética. Aquichan. 2009;9(2):171-84.

36. Herv A, Garc E. Situación cognitiva como condicionante de fragilidad en el anciano: perspectiva desde un centro de salud. An Sist Sanit Navar. 2005;28(1):35-47.

\section{Notas}

* $\quad$ Artigo de pesquisa.

\section{BY}

\section{Informação adicional}

Como citar: Coelho SP, Pinho-Reis C, Capelas ML, Sá L. Avaliação cognitiva de pacientes em cuidados paliativos: estudo piloto. Investig Enferm. Imagen Desarr. 2018;20(1):xx-xx. https://doi.org/10.11144/Jav eriana.ie20-1.acpc 